See discussions, stats, and author profiles for this publication at: https://www.researchgate.net/publication/330132331

\title{
Making use of Symmetries in the Three-Dimensional Elastic Inverse Homogenization Problem
}

Article in International Journal for Multiscale Computational Engineering · January 2019

DOI: $10.1615 /$ IntJMultCompEng.2019029111

CITATIONS

5 authors, including:

Carlos G. Mendez

Universidad Nacional del Litoral

22 PUBLICATIONS 105 CITATIONS

SEE PROFILE

Sebastian Toro

Universidad Nacional del Litoral

16 PUBLICATIONS 131 CITATIONS

SEE PROFILE

Some of the authors of this publication are also working on these related projects:

Project Fractional quantum Hall effect View project

Project Material design - Metamaterials View project
READS

1,251

Juan Manuel Podesta

National University of the Northeast

7 PUBLICATIONS 25 CITATIONS

SEE PROFILE

Alfredo E. Huespe

Universidad Nacional del Litoral

111 PUBLICATIONS 2,347 CITATIONS

SEE PROFILE 
International Journal for Multiscale Computational Engineering, 17(3):261-280 (2019),

Special Issue: Computational Multi-scale Modeling and

Design of New Engineering Materials"

Guest Editors: M. Pietrzyk, T.Burczynski, X. Oliver, A. Huespe,)

\title{
Making use of Symmetries in the Three-Dimensional Elastic Inverse Homogenization Problem
}

\author{
C. Méndez ${ }^{1}$, J.M. Podestá ${ }^{1}$, S. Toro ${ }^{1}$, A.E. Huespe ${ }^{1,2 *}$ J. Oliver ${ }^{2,3}$ \\ ${ }^{1}$ CIMEC-UNL-CONICET, Predio Conicet Dr Alberto Cassano, CP 3000 Santa Fe, Argentina \\ ${ }^{2}$ Centre Internacional de Metodes Numerics en Enyinyeria (CIMNE),Campus Nord UPC. \\ ${ }^{3}$ E.T.S dEnginyers de Camins, Canals i Ports, Technical University of Catalonia (Barcelona Tech) \\ Campus Nord UPC, Mòdul C-1, c/ Jordi Girona 1-3, 08034, Barcelona, Spain
}

\begin{abstract}
The objective of this paper is the design of three-dimensional elastic metamaterials with periodic microarchitectures. The microarchitectures of these materials are attained by following an inverse design technique jointly with an homogenization-based topology optimization algorithm.

In this context, we have particularly studied the connection between the symmetry of the material layout at the microscale of 3D periodic composites and the symmetry of the effective elastic properties. We have analyzed some possible Bravais lattices and space groups, which are typically associated with crystallography, to study the way in which the symmetries of these geometrical objects can be usefully used for the microarchitecture design of $3 \mathrm{D}$ elastic metamaterial.

Following a previous work of the authors for two-dimensional problems, we suggest adopting the design domain of the topology optimization problem coincident with the Wigner-Seitz cells of specific Bravais lattices having the same point group to that of the target elasticity tensor.

The numerical assessment described in this papers aims at the design of an extreme material. The solutions obtained with this procedure show that different composite microarchitectures emerge depending on the cell shape selection.
\end{abstract}

Keywords: elastic symmetry; three-dimensional homogenization-based topology optimization; Wigner-Seitz 3D cells, synthesis of elastic microtructures.

\footnotetext{
${ }^{*}$ Corresponding author. E-mail address: ahuespe@cimec.unl.edu.ar (A.E. Huespe).
} 
International Journal for Multiscale Computational Engineering, 17(3):261-280 (2019),

Special Issue: Computational Multi-scale Modeling and

Design of New Engineering Materials"

Guest Editors: M. Pietrzyk, T.Burczynski, X. Oliver, A. Huespe,)

\section{Introduction}

\subsection{Objective and problem motivation}

In this paper, we present a methodology aiming at the microarchitecture synthesis of three-dimensional periodic composites whose homogenized elastic properties are similar to stipulated effective elasticities.

The design of composites satisfying this requirement is a well-known problem in the literature. It has been clearly explained and described in the book by [1] and references cited therein. A number of contributions addressed to solve this problem have been posteriorly published which should be mentioned in the following. In particular, typical examples where this problem naturally arise, within the context of a larger design problem, can be seen in the papers by [2] and [3].

To reach this objective, we appeal to the symmetries characterizing the material layouts of composites at the microstructural level as well as to the symmetry characterizing the stipulated effective elasticities.

The symmetry notion is ubiquitous in the nature. In general, this notion results in an extremely important physical property. Particularly, some features of material responses can be predicted without resorting to experimentation or complicated calculations by only appealing to the geometrical symmetries at different length scales of the material structure.

\subsection{Microarchitecture design using symmetric topologies}

The microarchitecture synthesis of a periodic composite with prescribed target effective elastic properties can be though as an inverse design problem that is formulated by means of an homogenization-based topology optimization problem, such as proposed in the pioneering work by [4] and widely reported in the posterior literature ([5], [6], [7], [8], [9]). Nice microstructures have been obtained by [10] with this formulation and more recently by [11] in the context of three-dimensional microstructure designs.

In the present paper, we follow this approach aiming at the three-dimensional microarchitecture design of elastic metamaterials realized as biphasic composites.

A particular issue arising in this type of inverse homogenization-based design approach is the selection of the domain shape where the topology optimization problem is solved. In our opinion, this domain shape would result in a good selection if, as an outcome of the approach, the same domain is also a unit cell of the designed composite.

There are some aspects to be considered for selecting adequate cell shapes. For example, [7] reported that the cell size influences the obtained topology solutions. But, which is more important for the approach taken in the present contribution is 
International Journal for Multiscale Computational Engineering, 17(3):261-280 (2019),

Special Issue: Computational Multi-scale Modeling and

Design of New Engineering Materials"

Guest Editors: M. Pietrzyk, T.Burczynski, X. Oliver, A. Huespe,)

the fact that a significant number of material layout topologies could be hidden or unreachable for some frequently used cell shapes, typically cubes or right rectangular prisms in 3D ([12]). Furthermore, It has been shown that the realization of certain classes of composites, such as the Vigdergauz microstructures or the microstructures proposed by [10], could be promoted by enforcing some kind of material layout symmetry. These issues have been particularly studied in the $2 \mathrm{D}$ elastic material design context by the authors in previous contributions, see [3] and [13].

In view of these observations and following similar arguments to the ones given by [13], here, we propose to employ concepts taken from crystallography to define the cell shape. These concepts are intimately related to the symmetry properties of the crystal structures and the elastic target tensors. Thus, three-dimensional crystal symmetry properties, defined according to their point groups and space groups, are the primary information guiding the selection of the unit cell shape that is considered for solving the topology optimization algorithm.

Our proposal consists of taking the Wigner-Seits cell of a Bravais lattice that is compatible with the point group of the target elasticity tensor. Also, the material layout at the microcell could be enforced to satisfy the space group symmetry compatible with the same elasticity tensor. By adopting this approach, it can be guarantee that the homogenized elastic properties of the designed composite will have the same, or higher, symmetry than the target ones. This methodology is a generalization to three-dimensional problems of the one reported in [13] for twodimensional cases.

By using this approach in the three-dimensional problems, we notice a challenge that does not exist in the two-dimensional ones; there is not any crystal system space group guaranteeing the realization of a periodic composite with isotropic effective elastic properties. Thus, we test our proposal by designing the microarchitecture of an isotropic extreme material with different Wigner-Seitz cells. In this test, the topology optimization algorithm does not explicitly impose the isotropy constraint. The obtained microstructure results are evaluated with a criterion measuring the proximity to an effective isotropic response.

A brief description of the paper is next given. Section 2 presents a brief summary of the microarchitecture design methodology that we follow. This methodology can be seen as an inverse homogenization technique formulated as a topology optimization problem. Section 3 and Appendix A give a short description of crystal symmetries and their connection with the symmetries of the material elastic properties. Evidently, a full explanation regarding the crystal structure symmetries largely exceed the scope of the present paper; thus, the interested reader should consult the specific literature for additional information on this topic.

Section 4 describes the main contribution of the paper. We explain the proposed procedure guiding the selection of the cell shape and the possible space group symmetry to be imposed in the microarchitecture topology design. 
International Journal for Multiscale Computational Engineering, 17(3):261-280 (2019),

Special Issue: Computational Multi-scale Modeling and

Design of New Engineering Materials"

Guest Editors: M. Pietrzyk, T.Burczynski, X. Oliver, A. Huespe,)

A numerical assessment making use of this procedure is presented in Section 5. We show a three-dimensionl design problem of an extreme isotropic composite material with maximum shear and bulk modulus. We seek the solution by only testing a cubic crystal system and its three related Bravais lattices.

Finally, Section 6 presents the conclusions and final remarks of this paper. The topology optimization algorithm is briefly described in Appendix B.

\section{Homogenization-based topology optimization prob- lem}

Material design via inverse homogenization refers to the problem of finding the material configuration at the microscale of a periodic composite whose effective elasticity tensor is identical to a target elasticity tensor. This problem has been formulated as an homogenization-based topology optimization problem in the pioneering works of [4], [14] and [10]. In the following years, several works using this approach and emplying a variety of topology optimization algorithms have been reported. Only to mention a few, we cite the works by [5], [11] who describe threedimensionl elastic metamaterial designs using the SIMP optimization algorithm, and [15], who have used the bi-directional evolutionary structural optimization (BESO) algorithm. Two interesting recent reviews about this methodology can be found in [16] and [17]).

Here, we follow a similar homogenization-based approach. Let us consider a structure whose material is a periodic composite constituted by two isotropic elastic phases $M_{1}$ and $M_{2}$. We take a unit cell of this material identified by $\Omega_{\mu}$. In this microcell, phases $M_{1}$ and $M_{2}$ occupy the domains $\Omega_{\mu}^{1}$ and $\Omega_{\mu}^{2}$, respectively, see Figure 1.

The characteristic function $\chi(\boldsymbol{y})$ is defined in $\Omega_{\mu}$. It identifies the positions where the phase $M_{1}$ is placed and takes the following values:

$$
\chi(\boldsymbol{y})=\left\{\begin{array}{ll}
0 & \forall \boldsymbol{y} \in \Omega_{\mu}^{2} \\
1 & \forall \boldsymbol{y} \in \Omega_{\mu}^{1}
\end{array} .\right.
$$

Evidently, the homogenized elasticity tensor of the composite, $\boldsymbol{C}^{h}$, depends on the geometrical configuration of the phases $M_{1}$ and $M_{2}$ in $\Omega_{\mu}$. This dependence is made explicit by introducing the notation $\boldsymbol{C}^{h}(\chi)$. This tensor can be evaluated in $\Omega_{\mu}$ by enforcing periodic boundary conditions in displacements fluctuations. Then, standard computational techniques based on finite elements ([18]) or Fast Fourier Transform ([19]) can be used to get this goal. 
International Journal for Multiscale Computational Engineering, 17(3):261-280 (2019),

Special Issue: Computational Multi-scale Modeling and Design of New Engineering Materials"

Guest Editors: M. Pietrzyk, T.Burczynski, X. Oliver, A. Huespe,)

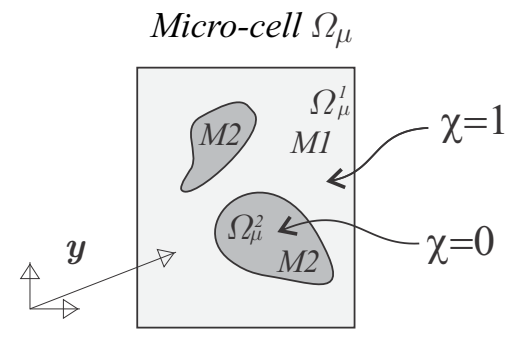

Figure 1: Microcell used as the design domain for the Topology Optimization Problem. The material layout is defined in $\Omega_{\mu}$. The homogenized elastic properties $C^{h}$ of the composites are also computed in $\Omega_{\mu}$

\subsection{The topology optimization algorithm}

Next, we formulate the microarchitecture inverse design problem as a topology optimization problem. Let be given the design domain $\Omega_{\mu}$ and the target effective elasticity tensor $\hat{\boldsymbol{C}}$. Let also be given the space $\mathcal{V}_{\chi}$ collecting together all the characteristic functions $\chi(\boldsymbol{y})$ in $\Omega_{\mu}$ where the domains $\Omega_{\mu}^{1}$ and $\Omega_{\mu}^{2}$ can be arbitrarily changed. Then, the optimization problem is formulated as follows:

$$
\begin{aligned}
\min _{\chi \in \mathcal{V}_{\chi}} & \left\|\boldsymbol{C}^{h}(\chi)-\hat{\boldsymbol{C}}\right\| \\
& \text { such that: } V^{h}(\chi)-V^{o b j}=0 .
\end{aligned}
$$

Where $V^{h}=\left(\int_{\Omega_{\mu}} \chi d \Omega\right) /\left|\Omega_{\mu}\right|$, is the volume fraction of the stiff phase $M_{1}$ and $V^{o b j}$ is the target volume fraction of the same phase.

The problem (2) can be reformulated and implemented by defining a continuous function $\psi$ in $\Omega_{\mu}$ whose zero level set $(\psi=0)$ identifies the stiff-void material interface. The position of the zero level set is iteratively updated, using a gradientlike method. The update direction for $\psi$ is defined through the topological derivative of $\boldsymbol{C}^{h}$. Hence, the problem (2) is rephrased as the optimization problem (13), in Appendix B. The algorithm for solving it has been reported by Amstutz and coworkers, see [20] and [21], and a brief summary is presented in Appendix B.

To facilitate the comparative analysis performed in Section 5, we use a conventional Helmholtz filter forcing the optimization algorithm in Appendix B to provide solutions displaying approximately only one length scale of the stiff phase. This filter is implemented using equation (25), Appendix B. Additional details of this filter can be found in [22] and [23].

\subsection{Issues related to the design domain selection}

Two implicit variables in the problem (13) of Appendix B must be defined in advance. Their values result from a decision taken by the designer and play an im- 
International Journal for Multiscale Computational Engineering, 17(3):261-280 (2019),

Special Issue: Computational Multi-scale Modeling and

Design of New Engineering Materials"

Guest Editors: M. Pietrzyk, T.Burczynski, X. Oliver, A. Huespe,)

portant role to govern the complexity of the attained microarchitecture topology. They are:

i) the shape of the design domain $\Omega_{\mu}$.

ii) the microstructure periodicity directions. In fact, to find the effective properties of the composite, it is necessary to impose constraints on the displacement fluctuations on the boundary of $\Omega_{\mu}$ which are compatible with the characteristic periodicity directions of the composite. The periodic constraints in the inverse homogenization problem result from a decision taken by the designer, by arguing that the microarchitecture is periodic along pre-established directions. Due to this assumption, the full material architecture is a spatial replica, by tessellation, of the cell $\Omega_{\mu}$.

\section{Crystal symmetry properties}

We start this Section by briefly discussing some crystal symmetry properties. In the present context of the microarchitecture design, crystal symmetries are exploited to guide the designer in making adequate decisions about the design domain selection.

Crystals can be characterized according to their specific symmetry properties. These properties are inherited from the underlying Bravais lattices and from their motifs. Two crystals sharing identical point group symmetry elements are said to belong to the same crystal system. Implicit to this classification is considered the translational and glide symmetries of the crystal motif which are taken into account to characterize the crystal symmetry properties, they constitute the space group of the crystal system. We postpone until Appendix A the discussion of further details about well-established crystal symmetry properties and the description of the notation adopted to identify point and space groups which are used in this Section ${ }^{1}$.

The characteristic feature referred to the crystal structure that is here stressed is the relationship between the crystal symmetry of a given material and the symmetry of its associated effective physical properties. In particular, the elastic symmetry properties. This relationship can be stated in terms of the Neumann's principle which establishes that: the symmetry elements of any physical property of a crystal must include the symmetry elements of the point group of the crystal. (see [26], pp.20).

According to this principle, the relation between crystal systems and elastic symmetry classes is given by their compatibility with similar point group transformations. This fact is summarized in Table 1 displaying six columns that are explained

\footnotetext{
${ }^{1}$ For a detailed mathematical and physical description of the following concepts: crystal system, Bravais lattice, lattice system, point group, space group, etc., the reader may consult [24] and [25].
} 
International Journal for Multiscale Computational Engineering, 17(3):261-280 (2019),

Special Issue: Computational Multi-scale Modeling and

Design of New Engineering Materials"

Guest Editors: M. Pietrzyk, T.Burczynski, X. Oliver, A. Huespe,)

in the next sub-Sections. Each row, in the first column, describes an elasticity tensor class which is associated with a specific crystal system and to more than one possible point groups and space groups. The fifth column indicates the number of Bravais lattice types that share identical point group symmetries with the elasticity class shown in column 1 . They are collected together as a lattice system. These Bravais lattices are depicted in Table 5, Appendix A.

\subsection{Elastic symmetry classes and crystal systems}

The first column in Table 1 displays the structure of the matrix representation ${ }^{2}$ of the elasticity tensor $\boldsymbol{C}$ and the identities that its coefficients have to satisfy accordingly to the symmetry of the elasticity class to which it belongs to.

The established relations between the first and third columns in Table 1 are determined with the procedure proposed by [27]. This procedure uses the following sequence of operations. First, a point group is taken. Subsequently, the symmetry conditions being compatible with the point group elements are enforced to the elastic response, and the resulting format of the elasticity matrix is determined. Following this procedure with the 32 point groups, it is possible to collect together the set of point groups rendering identical matrix formats. Thus, it can be concluded that every matrix format is compatible with a specific set of point groups.

The crystal symmetries can be classified by using a similar procedure, but now the cross relationships, i.e. finding common symmetry elements, are performed with the space group transformations. Using this technique, only seven crystal systems are determined.

Furthermore, as a consequence of the Neumann's principle, crystal systems and elasticity classes are related by sharing similar point groups. This association is evidenced in Table 1 between the first and second column. Therefore, an elastic class is designed with the name given to the corresponding crystal system. An exception to this rule is the isotropic elastic class which has not associated any crystal system.

The coefficients of the elastic matrices are described in a coordinate system whose coordinate planes coincide with the crystal symmetry planes. We call it the natural coordinate system. Additionally, by appealing to thermodynamic stability arguments, these matrices should be positive definite.

\footnotetext{
${ }^{2}$ Kelvin's notation is assumed for the matrix form of the elasticity tensor
} 
International Journal for Multiscale Computational Engineering, 17(3):261-280 (2019),

Special Issue: Computational Multi-scale Modeling and Design of New Engineering Materials"

Guest Editors: M. Pietrzyk, T.Burczynski, X. Oliver, A. Huespe,)

Table 1: Symmetry elements (point and space groups) of Crystal Systems (CS), Elasticity Classes (EC) and Lattice Systems (LS). Each row identifies the triplet (CS-EC-LS) with point group compatibility. First column describes the matrix format of the elasticity tensor class. The symbols in column 1 indicates: "*" a possibly non-zero component; " $*-*$ " two identical components; " $*-\bar{*}$ " two equal components with opposite sign; " $\otimes$ " the shear moduli is equal to $\left(C_{11}-C_{12}\right)$ (see Ting [27]). Fifth columns indicate the number of Bravais lattices constituting the lattice system defined in the sixth column.

\begin{tabular}{|c|c|c|c|c|c|}
\hline $\begin{array}{l}\text { Elasticity } \\
\text { Tensor }\end{array}$ & $\begin{array}{l}\text { Crystal } \\
\text { System }\end{array}$ & $\begin{array}{l}\text { Point } \\
\text { Groups }\end{array}$ & $\begin{array}{l}\text { Space } \\
\text { Groups }\end{array}$ & $\begin{array}{l}\text { Nbr. of Bra- } \\
\text { vais Lattices }\end{array}$ & $\begin{array}{l}\text { Lattice } \\
\text { System }\end{array}$ \\
\hline$\left(\begin{array}{cccccc}* & * & * & * & * & * \\
& * & * & * & * & * \\
& & * & * & * & * \\
& & & * & * & * \\
& & & & * & * \\
& & & & *\end{array}\right)$ & Triclinic & $1, \overline{1}$ & 2 & 1 & Triclinic \\
\hline 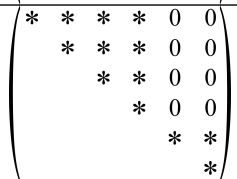 & Monoclinic & $2, \mathrm{~m}, 2 / \mathrm{m}$ & 13 & 2 & Monoclinic \\
\hline 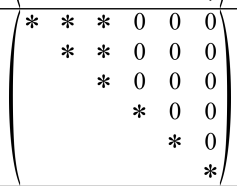 & $\begin{array}{l}\text { Ortho- } \\
\text { rhombic }\end{array}$ & $\begin{array}{l}222, \mathrm{~mm} 2 \\
\mathrm{mmm}\end{array}$ & 59 & 4 & $\begin{array}{l}\text { Ortho- } \\
\text { rhombic }\end{array}$ \\
\hline 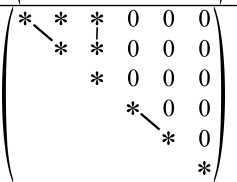 & Tetragonal & $\begin{array}{c}4, \overline{4}, 4 / \mathrm{m}, \\
442,4 \mathrm{~mm}, \\
\overline{4} 2 \mathrm{~m}, 4 / \mathrm{mmm}\end{array}$ & 68 & 2 & Tetragonal \\
\hline 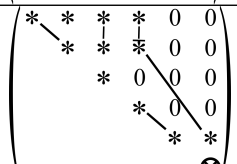 & Trigonal & $\begin{array}{c}3, \overline{3} \\
32,3 \mathrm{~m}, \\
\overline{3} \mathrm{~m}\end{array}$ & 7 & 1 & $\begin{array}{l}\text { Rhombo- } \\
\text { hedral }\end{array}$ \\
\hline & & & 18 & \multirow{2}{*}{1} & \multirow{2}{*}{ Hexagonal } \\
\hline$\left(\begin{array}{cccccc}* & * & * & 0 & 0 & 0 \\
& * & * & 0 & 0 & 0 \\
& & * & 0 & 0 & 0 \\
& & & * & 0 & 0 \\
& & & & * & 0 \\
& & & & 8\end{array}\right)$ & Hexagonal & $\begin{array}{c}6, \overline{6}, \\
6 / \mathrm{m}, 622, \\
6 \mathrm{~mm}, \overline{6} \mathrm{~m} 2, \\
6 / \mathrm{mmm}\end{array}$ & 27 & & \\
\hline$\left(\begin{array}{ccccc}* & *-* & 0 & 0 & 0 \\
*_{*}^{*} & * & 0 & 0 & 0 \\
& * & 0 & 0 & 0 \\
& & * & 0 & 0 \\
& & & * & 0 \\
& & & & *\end{array}\right)$ & Cubic & $\begin{array}{c}23, \mathrm{~m} \overline{3} \\
432, \overline{4} 3 \mathrm{~m}, \\
\mathrm{~m} \overline{3} \mathrm{~m}\end{array}$ & 36 & 3 & Cubic \\
\hline Total & 7 & 32 & 230 & 14 & 7 \\
\hline
\end{tabular}


International Journal for Multiscale Computational Engineering, 17(3):261-280 (2019),

Special Issue: Computational Multi-scale Modeling and

Design of New Engineering Materials"

Guest Editors: M. Pietrzyk, T.Burczynski, X. Oliver, A. Huespe,)

\section{Microarchitecture design tools based on the tar- get elastic symmetry}

The characteristic symmetries of crystal structures described in Table 1 and complemented with the information presented in Table 5, Appendix A, provide the basis for guiding the selection of the domain shape $\Omega_{\mu}$ that is described in Section 2 . The information in these Tables is used to define the next procedure.

1) The domain $\Omega_{\mu}$, which is used for solving the topology optimization problem (2), is the Wigner-Seitz cell of a Bravais lattice that belongs to the lattice system whose point group is compatible with the target elasticity tensor symmetry.

This compatibility relationship between lattice point group and elastic symmetry is implicit in Table 1 because the elasticity classes and the Bravais lattice systems with compatible point groups are placed in identical rows. Consequently, the Table indicates an automatic procedure to define the cell domain by choosing a Bravais lattice of the corresponding lattice system. Note that the specific descriptions of the 14 three-dimensional lattices that belong to different lattice systems are represented in Table 5 of Appendix A.

For example, let us consider a target elasticity tensor with hexagonal symmetry. This elastic symmetry class is classified in the sixth row of Table 1 and is compatible with only one Bravais lattice having a point group $6 / \mathrm{mmm}$. This lattice is displayed in the sixth row of Table 5. According to the present proposal, the design domain $\Omega_{\mu}$ for solving the problem (2) should be similar to the Wigner-Seitz cell of this compatible lattice. This Wigner-Seitz cell is schematized in Figure 2.

We remark that the periodicity directions of the microstructure to be designed are implicitly defined once the Wigner-Seitz cell is adopted because they coincide with the Bravais lattice primitive directions.

2) Furthermore, the procedure can guarantee beforehand that the homogenized elasticity tensor of the designed composite will preserve the same symmetry class to that of the target one. This feature is achieved if the solution of the optimization algorithm is constrained to attain a symmetric topology compatible with the space group of the elasticity tensor class. Taken the same example above mentioned, a target tensor with hexagonal symmetry, we can guarantee that the homogenized elasticity of the designed composite will also have hexagonal symmetry if the solved topology is compatible with the space group $P 6 / \mathrm{mmm}$. 
International Journal for Multiscale Computational Engineering, 17(3):261-280 (2019),

Special Issue: Computational Multi-scale Modeling and Design of New Engineering Materials"

Guest Editors: M. Pietrzyk, T.Burczynski, X. Oliver, A. Huespe,)

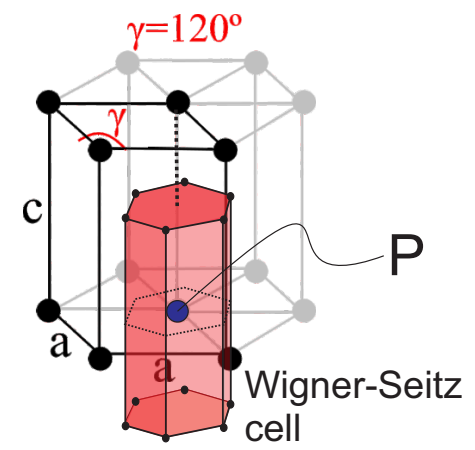

Figure 2: Wigner-Seitz cell (in red color) of the primitive hexagonal Bravais lattice whose point group is $6 / \mathrm{mmm}$. Atom $\mathrm{P}$ is the Voronaoi cell center.

\subsection{Cubic system}

A particular interest in metamaterial design aims at attaining microarchitectures with effective isotropic elastic properties. Such as mentioned above, the isotropic elasticity class is not represented in Table 1 because there is not a crystal system whose compatible point group determines this class of elastic symmetry.

Due to this feature, we focus our attention in the cubic crystal system to design isotropic metamaterials. This crystal system shows the higher possible symmetry of all crystal structures. Table 2 describes additional details of the properties associated with the Cubic System which are used in the next numerical assessment. They can be summarized as follows:

i) There are three Bravais lattices compatible with the cubic system: Primitive, Face Centered and Body-Centered, see Table 5 of Appendix A. They are denoted SC, FCC, and BCC, respectively.

ii) Five-point groups and 36 spatial groups are compatible with the Cubic system.

Spatial groups of these lattices with high symmetries are $P m \overline{3} m, F m \overline{3} m, \operatorname{Im} \overline{3} m$ which share the point group $m \overline{3} m$ guaranteeing the cubic symmetry of the effective crystal elastic properties. These spatial groups are chosen in this paper to define the initial configurations of the iterative algorithm used to solve the numerical test presented in next sub-Section.

Figure 3-a depicts some of the point group symmetry elements of the cubic system (BCC). A crystal motif is introduced in Figure 3-b, therefore, the symmetry element $\overline{3}$ belongs to the space group $P m \overline{3} m$.

Table 2 additionally depicts the three Wigner-Seitz cells of the three cubic lattices. These unit-cells preserve the point group symmetries of the underlying Bravais lattices. 
International Journal for Multiscale Computational Engineering, 17(3):261-280 (2019),

Special Issue: Computational Multi-scale Modeling and Design of New Engineering Materials"

Guest Editors: M. Pietrzyk, T.Burczynski, X. Oliver, A. Huespe,)

Table 2: Cubic System. Compatible point and space groups. Bravais lattices compatible with the cubic systems are: Primitive (SC), Face Centered Cubic (FCC) and Body Centered Cubic (BCC). Weigner-Seitz cells of the three Bravais lattices.

\begin{tabular}{|c|c|c|c|c|c|}
\hline $\begin{array}{l}\text { Elasticity } \\
\text { Tensor }\end{array}$ & $\begin{array}{l}\text { Crystal } \\
\text { System }\end{array}$ & $\begin{array}{l}\text { Point } \\
\text { Groups }\end{array}$ & \multicolumn{3}{|c|}{$\begin{array}{l}\text { Space } \\
\text { Groups }\end{array}$} \\
\hline \multirow{5}{*}{$\left.\begin{array}{ccccc}* & 0 & 0 & 0 \\
& * & 0 & 0 \\
& & & * & 0 \\
& & & & *\end{array}\right]$} & \multirow{5}{*}{ Cubic } & 23 & $\mathrm{P} 23, \mathrm{P} 2{ }_{1} 3$ & F23 & $\mathrm{I} 23, \mathrm{I} 2{ }_{1} 3$ \\
\hline & & $\mathrm{m} \overline{3}$ & $\mathrm{Pm} \overline{3}, \mathrm{Pn} \overline{3}, \mathrm{~Pa} \overline{3}$ & $\mathrm{Fm} \overline{3}, \mathrm{Fd} \overline{3}$ & $\mathrm{I} \overline{3}, \mathrm{Ia} \overline{3}$ \\
\hline & & 432 & $\begin{array}{l}\mathrm{P} 432, \mathrm{P} 4_{2} 32, \\
\mathrm{P} 4_{3} 32, \mathrm{P} 4_{1} 32\end{array}$ & $\mathrm{~F} 432, \mathrm{~F} 4_{1} 32$ & $\mathrm{I} 432, \mathrm{I}_{1} 32$ \\
\hline & & $\overline{4} \overline{3 m}$ & $\mathrm{P} \overline{4} 3 \mathrm{~m}, \mathrm{P} \overline{4} 3 \mathrm{n}$ & $\mathrm{F} \overline{4} 3 \mathrm{~m}, \mathrm{~F} \overline{4} 3 \mathrm{c}$ & $\mathrm{I} \overline{4} 3 \mathrm{~m}, \mathrm{I} \overline{4} 3 \mathrm{~d}$ \\
\hline & & $\mathbf{m} \overline{\mathbf{3}} \mathbf{m}$ & $\begin{array}{c}\mathbf{P m} \overline{\mathbf{3}} \mathbf{m}, \operatorname{Pn} \overline{3} n \\
\operatorname{Pm} \overline{3} n, \operatorname{Pn} \overline{3} m\end{array}$ & $\begin{array}{c}\mathbf{F m} \overline{\mathbf{3}} \mathbf{m}, \operatorname{Fm} \overline{3} \mathrm{c}, \\
\mathrm{Fd} \overline{3} \mathrm{~m}, \mathrm{Fd} \overline{3} \mathrm{c}\end{array}$ & $\operatorname{Im} \overline{\mathbf{3}} \mathbf{m}$, Ia $\overline{3} \mathrm{~d}$ \\
\hline \multicolumn{3}{|c|}{ Compatible Bravais lattice } & & & \\
\hline \multicolumn{3}{|c|}{ Wigner-Seitz cell } & & & \\
\hline
\end{tabular}
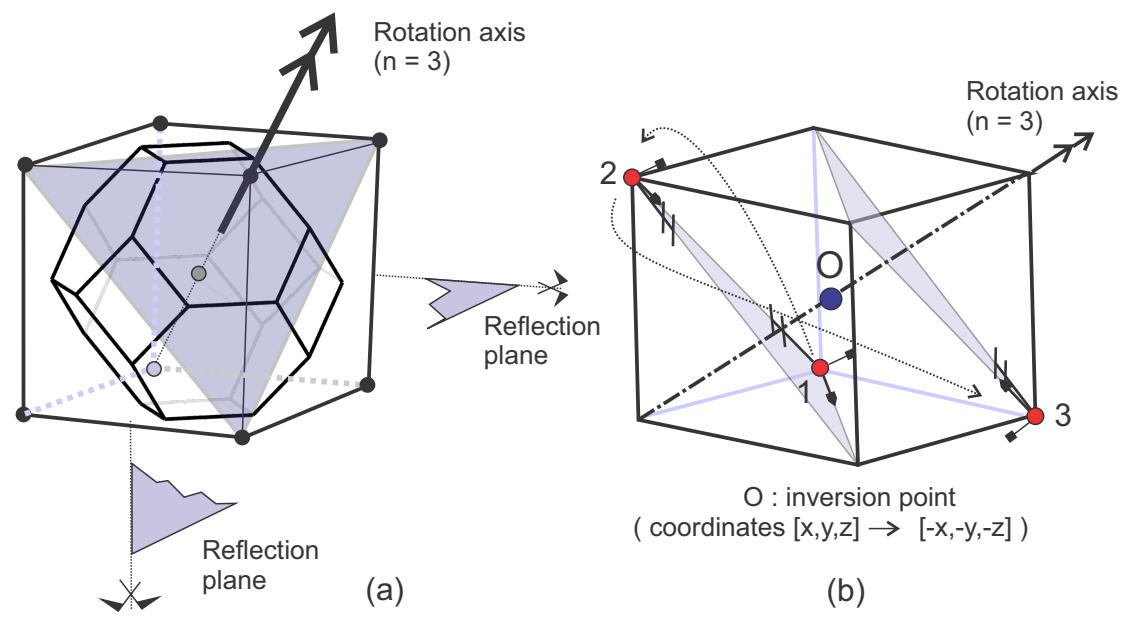

(b)

Figure 3: a) Some point group elements of the BCC system. A threefold rotoinversion and two reflection planes. The Wigner-Seitz cell preserves these point group symmetry elements. b) Representation of the typical cubic system symmetry element $\overline{3}$. The symbol $\overline{3}$ indicates that the crystal pattern will remain identical after a $\pi / 3[\mathrm{rad}]$ rotation (transformation $1 \rightarrow 2$ ) followed by an inversion through the atom $\mathrm{O}$ (transformation $2 \rightarrow 3$ ). 


\section{Design of an extremal isotropic material. $\mathrm{Nu}-$ merical application.}

We design the three-dimensionl microarchitecture of a metamaterial constituted of void and a stiff phase. The objective is to attain an isotropic composite displaying maximum effective shear and bulk moduli, $G^{e f f}$ and $K^{e f f}$, respectively, for a given volume fraction of the stiff phase. A similar topology design problem has been solved and reported in the literature using the SIMP technique by [11] and the BESO technique by [28]. Here, we particularly study the microarchitecture topologies obtained with the present proposal, as well as, the closeness to elastic isotropy of the designed composite overall response.

[29] have reported bounds for the maximum and minimum effective attainable shear and stiffness moduli of two-phase composites with (quasi-) isotropic overall properties. In particular, for a composite constituted of void and a stiff phase with bulk modulus $K$, shear modulus $G$ and volume fraction $f, K^{e f f}$ and $G^{e f f}$ satisfy:

$$
\begin{gathered}
K^{e f f} \leq \frac{4 G K f}{4 G+3 K(1-f)}, \\
G^{f f} \leq G+\frac{1-f}{\frac{6}{5 G} \frac{(K+2 G) f}{(3 K+4 G)}-\frac{1}{G}} .
\end{gathered}
$$

The right hand part of these expressions are the Hashin and Shtrikman upperbounds.

Stiff phase material properties. In the present design problem, the values of bulk and shear moduli of the stiff phase are $K=1.669[G P a]$ and $G=0.3571[G P a]$. The void is numerically simulated by taking a contrast factor $\gamma=1 e-5$, being $\gamma$ the ratio between an assumed pseudo-void moduli and the stiff phase moduli. The stiff phase void fraction is $f=0.338$.

Target elasticity tensor for the inverse design problem: Figure 4 displays the Hashin-Strikman upper-bounds defined by the right hand terms of the expressions (3) and (4). These bounds are plotted in the space $\left(K^{e f f}, G^{e f f}\right)$ with green lines. The point with maximum shear and bulk moduli at the top-right vertex of these bounds is shown in the plot with the green point and coordinates $\hat{K}=0.1699[G P a]$ and $\hat{G}=0.0789[G P a]$. This point represents the properties of the target extreme metamaterial that is designed in the present assessment. In Kelvin notation, the corresponding target isotropic elastic tensor, $\hat{C}$, of an isotropic material whose bulk and shear modulus are $\hat{K}$ and $\hat{G}$ is given by : 
International Journal for Multiscale Computational Engineering, 17(3):261-280 (2019),

Special Issue: Computational Multi-scale Modeling and

Design of New Engineering Materials"

Guest Editors: M. Pietrzyk, T.Burczynski, X. Oliver, A. Huespe,)

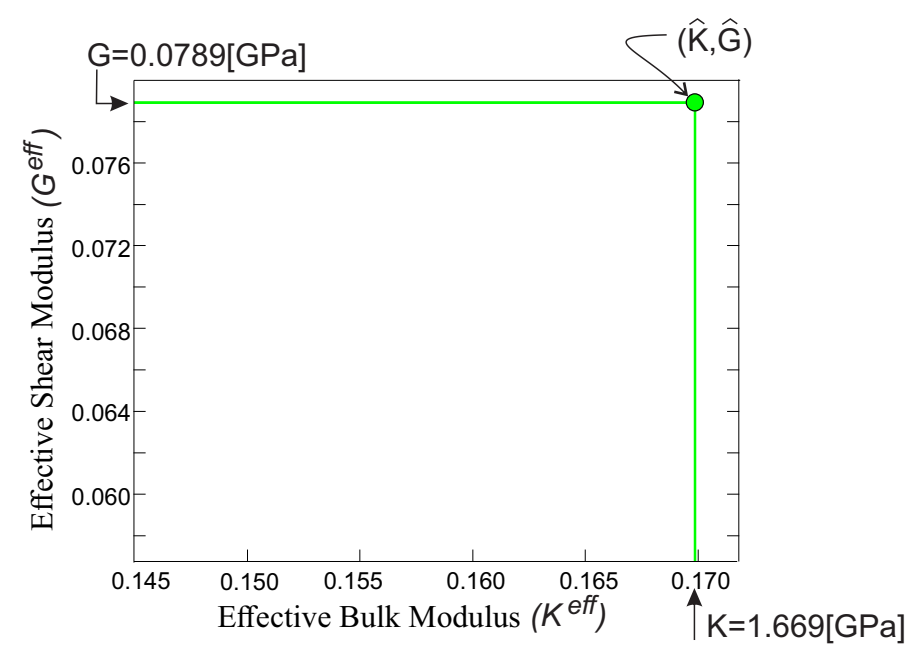

Figure 4: Hashin-Strikman upper-bounds in the space $\left(K^{e f f}, G^{e f f}\right)$.

$$
\hat{C}=\left(\begin{array}{ccccccc}
\hat{K}+\frac{4}{3} \hat{G} & \hat{K}-\frac{2}{3} \hat{G} & \hat{K}-\frac{2}{3} \hat{G} & 0 & 0 & 0 \\
& \hat{K}+\frac{4}{3} \hat{G} & \hat{K}-\frac{2}{3} \hat{G} & 0 & 0 & 0 \\
& & \hat{K}+\frac{4}{3} \hat{G} & 0 & 0 & 0 \\
& & & 2 \hat{G} & 0 & 0 \\
& & & & & 2 \hat{G} & 0 \\
& & & & & 2 \hat{G}
\end{array}\right)
$$

\subsection{Algorithm and cells for solving the optimization prob- lem}

The tensor $\hat{C}$ of expression (5) and the constraint imposed on the stiff phase volume fraction are the data inputs defining the topology optimization problem (13).

The sensitivities of the microarchitecture solutions with the adopted cell shapes are estimated. Thus, we take three different classes of cells which are the WignerSetiz cells of the following Bravais lattices that are compatible with the Cubic lattice system:

- Primitive Cubic Bravais Lattice (SC)

- Body Centered Cubic Bravais Lattice (BCC)

- Face Centered Cubic Bravais Lattice (FCC)

These Bravais Lattices and the corresponding Wigner-Seitz cells are shown in Table 3. Here, we restrict the numerical evaluations to test Cubic system lattices because they have the higher symmetry. The lattice parameter of the three cubic lattices is $a=1$. To attain comparable results, we define the volumes of the SC, BCC and 
International Journal for Multiscale Computational Engineering, 17(3):261-280 (2019),

Special Issue: Computational Multi-scale Modeling and Design of New Engineering Materials"

Guest Editors: M. Pietrzyk, T.Burczynski, X. Oliver, A. Huespe,)

Table 3: Cells compatible with the Cubic lattice systems used in the numerical tests

\begin{tabular}{|c|c|c|}
\hline Lattice System & Bravais Lattice Wigner-Seitz Cell \\
\hline $\begin{array}{c}\text { Bedy centered } \\
\text { BCC }\end{array}$ \\
Face centered \\
FCC
\end{tabular}

FCC Wigner-Seitz cells as 1, 0.5 and 0.25, respectively. A property of the WignerSeitz cells is that their volumes are proportional to the number of atoms per cell in the corresponding lattice systems.

The algorithm does not enforce the microarchitecture topologies to be compatible with a specific space group symmetry. Therefore, the obtained topologies and the overall symmetry properties of the designed composites will be strictly related to the specific cells used in each test.

The optimization algorithm uses a Helmholtz filter with the parameter $r_{l s}=$ 0.001 , see equation (25) of Appendix B. This filter prescribes a minimum section of the stiff phase that could be achieved in the material layout solutions. Therefore, the microarchitectures will display topologies with approximately one length scale. Additional discussion on this topic can be found in [22].

Finite element models of the cells. The finite elements are linear tetrahedrons. We only mesh a reduced domain of the Wigner-Seitz cells. These reduced zones are depicted in the Figure 5 for the FCC and BCC cells and are obtained following a similar procedure to get the irreducible Brillouin zones. Subsequently, the mesh of the full Wigner-Seitz cell is generated by applying successive point symmetry operations (reflections and rotations) to the original reduced zone mesh. 
International Journal for Multiscale Computational Engineering, 17(3):261-280 (2019),

Special Issue: Computational Multi-scale Modeling and Design of New Engineering Materials"

Guest Editors: M. Pietrzyk, T.Burczynski, X. Oliver, A. Huespe,)

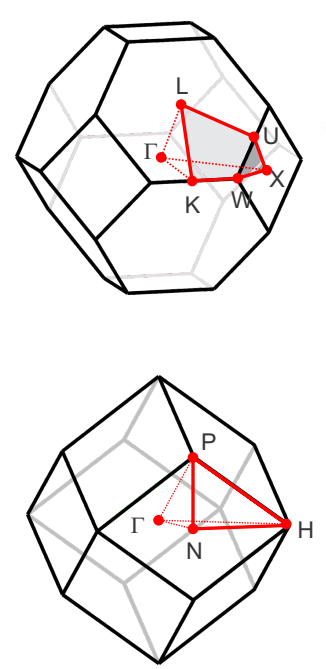

(a)
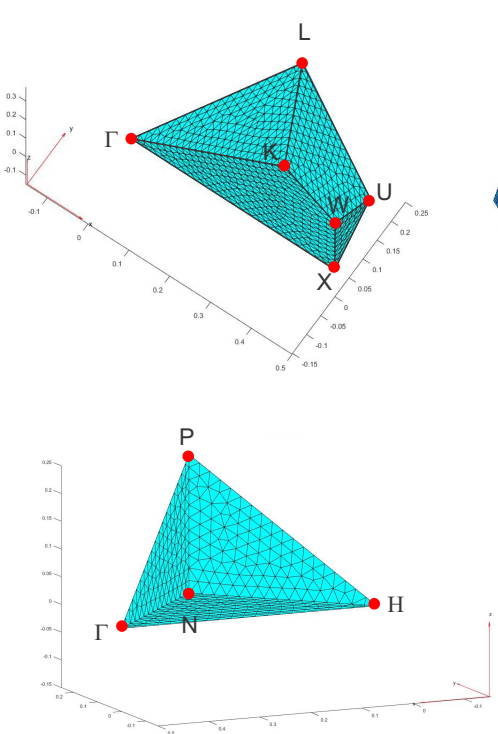

(b)
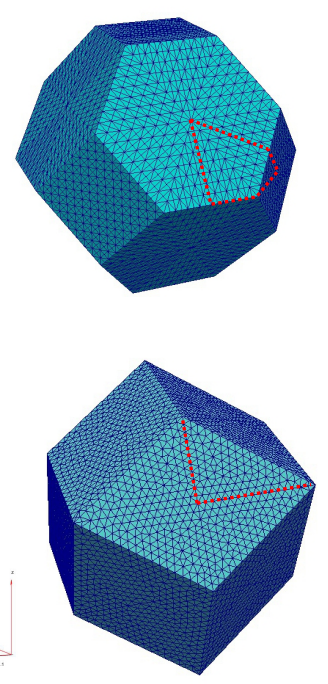

(c)

Figure 5: Finite element meshes of the Wigner-Seitz cells (BCC and FCC cells). a) Reduced domains. $\Gamma$ is the central point of the Wigner-Seitz cell; $L$ the central point of a hexagonal face; $\mathrm{X}$ the central point of the contiguous square face; $\mathrm{K}$ the middle point of the edge between two hexagonal faces; $U$ the middle point of the edge between the hexagonal and square. a) Meshes of the reduced domains. b) Meshes of the full domains obtained from the meshes of the reduced domains by successive transformations of symmetry (reflections and rotations).

This meshing technique provides a symmetric finite element mesh that preserves the higher point group symmetry of the corresponding Wigner-Seitz cell. Thus, using these symmetric meshes, different kind of material distributions, with predetermined space group symmetries, can be easily implemented in the topology optimization algorithm.

using the so-generated symmetric meshes, different kind of material distributions, with predetermined space group symmetries, are much simpler to implement or impose in the topology optimization algorithm.

The number of finite elements in the three cells are taken such that the volume of the elements is similar in the three cases. Thus, the number of finite element in the SC, BCC and FCC cells are proportional to 1, 0.5 and 0.25 , respectively.

Starting configuration of the topology optimization algorithm. Topology optimization problems aiming at microstructure design, in general, contain many local minima. This characteristic induces a strong tendency to attain different solutions, depending on the initial guess configuration ([17]). Consequently, it is important to test several starting configurations to evaluate and compare the so-obtained 
International Journal for Multiscale Computational Engineering, 17(3):261-280 (2019),

Special Issue: Computational Multi-scale Modeling and Design of New Engineering Materials"

Guest Editors: M. Pietrzyk, T.Burczynski, X. Oliver, A. Huespe,)

solutions.

In the present numerical assessment, three initial configurations are tested: a) a spherical void placed around the central point of the cell; b) a cellular-like configuration with closed walls. The walls are coincident with the cell faces and the walls are of uniform thickness; c) a truss-like configuration with bars of identical sections joining the Wigner-Seitz cell vertices.

\subsection{Assessment of the cell capacity for capturing isotropic responses}

As already mentioned above, the isotropic elastic response of the designed composite cannot be guaranteed by only enforcing a stiff phase material layout consistent with the space group of some particular crystal system. This aspect of the threedimensionl problem introduces a marked difference with respect to the $2 \mathrm{D}$ case which has been analyzed in the previous contribution by the authors, see [13].

In consequence, we introduce an indicator to find how close are the homogenized properties of the designed composites to isotropic responses. Based on this indicator and without imposing implicitly the isotropy constraint into the formulation of the optimization algorithm, we assess which cell provides a better response to capture this elastic feature.

This indicator is computed as follows. Given an arbitrary elasticity tensor $\boldsymbol{C}^{h}$, we define an isotropic elastic tensor of comparison by following the procedure proposed by [30]. First, using the components of $\boldsymbol{C}^{h}$, it is evaluated:

$$
\begin{gathered}
C_{11}^{i s o}=\frac{1}{5}\left(C_{11}^{h}+C_{22}^{h}+C_{33}^{h}\right)+\frac{2}{15}\left(C_{12}^{h}+C_{13}^{h}+C_{23}^{h}\right)+\frac{4}{15}\left(C_{44}^{h}+C_{55}^{h}+C_{66}^{h}\right) \\
G^{i s o}=\frac{1}{15}\left(C_{11}^{h}+C_{22}^{h}+C_{33}^{h}\right)-\left(C_{12}^{h}+C_{13}^{h}+C_{23}^{h}\right)+3\left(C_{44}^{h}+C_{55}^{h}+C_{66}^{h}\right) \\
K^{i s o}=C_{11}^{i s o}-\frac{4}{3} G^{i s o}
\end{gathered}
$$

and with the so-determined bulk and shear moduli, $K^{i s o}$ and $G^{i s o}$, an isotropic tensor $\boldsymbol{C}^{\text {iso }}$ is computed with expression (5). Our assumption is that this is the closer isotropic tensor to the original $\boldsymbol{C}^{h}$. It is taken as the reference tensor to perform the following analysis.

Finally, we introduce a coefficient of anisotropy

$$
\chi=\left\|C^{h}-C^{i s o}\right\|
$$

which measures the distance between $C^{h}$ and $C^{i s o}$. A zero value of this coefficient indicates that $C^{h}$ is isotropic. Contrarily, a large value of $\chi$ indicates that $C^{h}$ is far from being isotropic. 
International Journal for Multiscale Computational Engineering, 17(3):261-280 (2019),

Special Issue: Computational Multi-scale Modeling and Design of New Engineering Materials"

Guest Editors: M. Pietrzyk, T.Burczynski, X. Oliver, A. Huespe,)

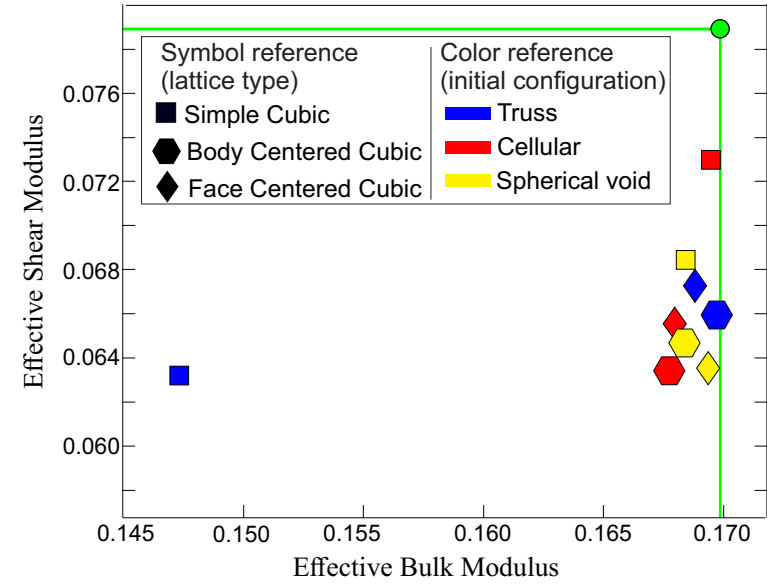

(a)

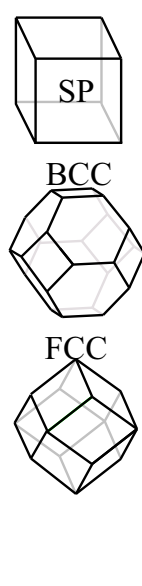

Figure 6: a) Space $K^{e f f}$ vs. $G^{e f f}$ : the results obtained with Primitive (SP), Face Centered (FFC) and Body-Centered (BCC) cubic Wigner-Seitz cells are superposed to the Hashin-Strikman upper bounds displayed in green lines. The initial configurations adopted in each case are distinguished with blue, red and yellow colors; b) Anisotropy coefficients $\chi$ for the nine tested case.

\subsection{Analysis of results}

Figure 6-a plots the results for the nine different cases in the space $K^{e f f}$ vs. $G^{e f f}$. They are compared with the upper Hashin-Strikman bounds, also plotted in the same Figure. The results have been computed using the three Wigner-Seitz cells: Primitive Cubic (SP), Face Centered Cubic (FFC) and Body-Centered Cubic (BCC) and the three initial configurations. The symbols identify the cell types and the colors identify the initial configurations.

The corresponding coefficients of anisotropy, defined by expression (9), are plotted in Figure 6-b. Independently of the adopted initial configurations, we note from this plot that the BCC cell provides microarchitecture topologies whose effective elastic properties tend to be more isotropic respect to the solutions provided by the other two alternative cells.

Figure 7 depicts the material distributions obtained with these cells and with the spherical void initial configurations. Two views of each solutions are displayed; the full cell solutions are shown in Figures b), d) and f) and the cells cut with middle planes are shown in Figures a), c) and e). Note that the material distributions assimilate to hollow topologies. Also, note the highly symmetric pattern of the material layouts obtained with the algorithm even without imposing any symmetry constraint.

Table 4 shows the components of the target effective elasticity tensor computed with expression (5). These components are compared with the homogenized tensor 
International Journal for Multiscale Computational Engineering, 17(3):261-280 (2019),

Special Issue: Computational Multi-scale Modeling and Design of New Engineering Materials"

Guest Editors: M. Pietrzyk, T.Burczynski, X. Oliver, A. Huespe,)

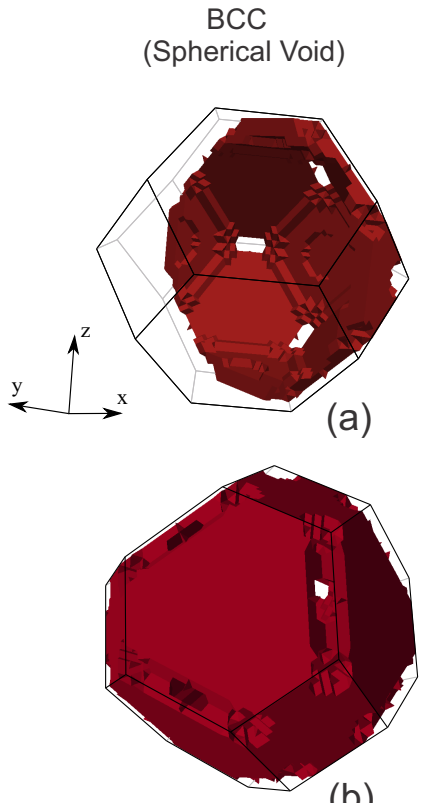

(b)

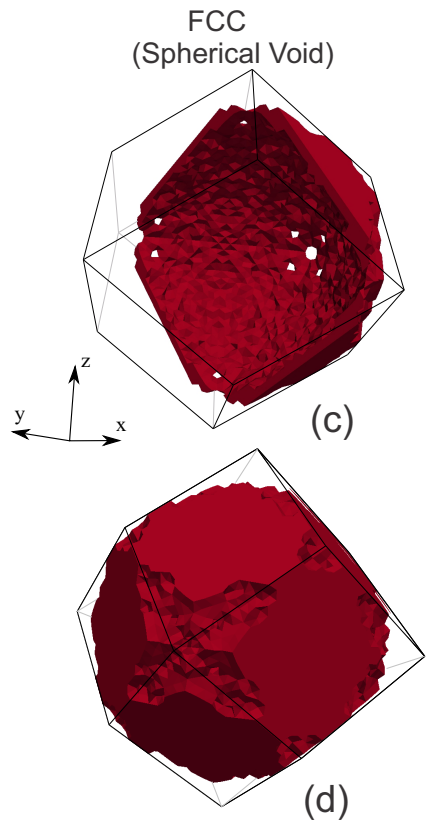

(d)
SP

(Spherical Void)

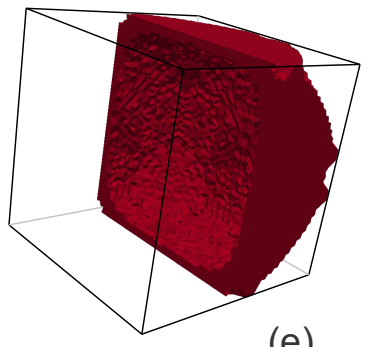

(e)

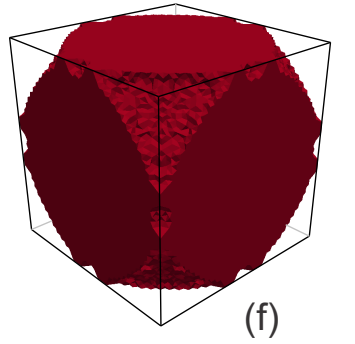

Figure 7: Microarchitectures with maximun bulk and shear moduli: a) and b) correspond to BCC cells (cutted with a middle plane (a) and full cell solutions (b), respectively); c) and d) correspond to FCC cells (cutted with a middle plane (c) and full cell solutions (d), respectively); e) and f) correspond to SP cells (cutted with a middle plane (e) and full cell solutions (f), respectively). 
International Journal for Multiscale Computational Engineering, 17(3):261-280 (2019),

Special Issue: Computational Multi-scale Modeling and

Design of New Engineering Materials"

Guest Editors: M. Pietrzyk, T.Burczynski, X. Oliver, A. Huespe,)

Table 4: Computed effective elasticity tensors

\begin{tabular}{|c|c|c|c|c|c|c|c|c|c|}
\hline & $C_{11}$ & $C_{22}$ & $C_{33}$ & $C_{44}$ & $C_{55}$ & $C_{66}$ & $C_{12}$ & $C_{13}$ & $C_{23}$ \\
\hline Target & 0.2750 & 0.2750 & 0.2750 & 0.1578 & 0.1578 & 0.1578 & 0.1172 & 0.1172 & 0.1172 \\
\hline $\begin{array}{c}\text { SP } \\
\text { Cellular }\end{array}$ & 0.2732 & 0.2732 & 0.2732 & 0.1398 & 0.1394 & 0.1392 & 0.1177 & 0.1177 & 0.1179 \\
\hline $\begin{array}{c}\text { BCC } \\
\text { Cellular }\end{array}$ & 0.2559 & 0.2571 & 0.2552 & 0.1272 & 0.1274 & 0.1276 & 0.1246 & 0.1243 & 0.1244 \\
\hline $\begin{array}{c}\text { FCC } \\
\text { cellular }\end{array}$ & 0.2570 & 0.2572 & 0.2569 & 0.1286 & 0.1274 & 0.1284 & 0.1242 & 0.1239 & 0.1241 \\
\hline
\end{tabular}

values obtained with the SP, BCC and FCC cells and cellular-like initial configurations. We observe from Figure 6-a that, apparently, the SP solution display closer values to the target ones. However, the identity $\hat{C}_{33}=\left(\hat{C}_{11}-\hat{C}_{12}\right)$, which must be satisfied by isotropic tensors, is more tightly verified with the BCC and FCC solutions if compared with the SP solution. This behavior, which is not transparent from results in Figure 6-a, is confirmed with those depicted in Figure 6-b. The stiff phase volume fractions in these solutions are accurate to the third figure $(f=0.338)$.

\section{Conclusions}

New contributions for synthesizing three-dimensionl microarchitectures of elastic composites are proposed. These contributions are addressed to enlarge the range of attainable microarchitectures in the framework of the inverse design problems formulated as homogenization-based topology optimization algorithms

Based on the symmetry of the target elastic properties, we propose a procedure for selecting a spatial three-dimensionl domain where the topology optimization algorithm is solved. Furthermore, this procedure also provides a route to enforce the symmetry of the material layout within this spatial domain compatible with predefined crystal spatial groups. The so-proposed rules are derived from concepts widely developed in crystallography. This contribution is a generalization of a procedure that has been previously presented by the authors in 2D problems.

The procedure has been tested by synthesizing an isotropic elastic material with prescribed maximum effective shear and bulk moduli. The results, which have been obtained without implicitly imposing a constraint of overall isotropic response, show that the $\mathrm{BCC}$ cells provide the tighter isotropic solution if compared with alternative cubic cells, no matter the initial configuration adopted for the topology optimization algorithm. Furthermore, by considering that the volume ratio between the SC and 
International Journal for Multiscale Computational Engineering, 17(3):261-280 (2019),

Special Issue: Computational Multi-scale Modeling and Design of New Engineering Materials"

Guest Editors: M. Pietrzyk, T.Burczynski, X. Oliver, A. Huespe,)

BCC cells is two; then, the size of the problem with the BCC cell is half of the problem size using the SC cell for similar resolutions.

An additional advantage of the proposed methodology is that very simple and precise numerical techniques, widely reported in the literature, can be used to generate the three-dimensionl Wigner-Seitz cells. Algorithms for determining the WignerSeitz cells of arbitrary distributions of points in the space, particularly the Bravais lattice atoms, are well developed in Computational Mechanics and can be easily used to determine the Wigner-Seitz cells of the 14 Bravais lattice types. Additionally, the technique based on meshing a reduced domain, and subsequently generate the mesh of the full cell through space group symmetry operations, facilitates the implementation of different types of symmetry constraints on the material layout.

\section{Acknowledgment}

The authors acknowledge the financial support from CONICET and ANPCyT (grants PICT 2014-3372 and 2016-2673).

Authors A.E. Huespe and J. Oliver acknowledge the funding received from the Spanish Ministry of Economy and Competitiveness through the research grant DPI2017-85521-P, Project "Computational design of Acoustic and Mechanical Metamaterials" (METAMAT).

\section{A APPENDIX I: Structure and Symmetry of Crys- tals}

\section{A.1 Bravais lattices}

Crystals are periodic structures, with periodicity along three linearly independent directions. This property is formalized by introducing the concept of a lattice. Lattices are defined by three vectors $\mathbf{a}, \mathbf{b}$ and $\mathbf{c}$, the primitive vectors of the lattice, which form a basis in $\mathbb{R}^{3}$. The set of abstract points, or atoms

$$
\mathbf{L}:=\{l \mathbf{a}+m \mathbf{a}+n \mathbf{c} \mid l, m, n \in \mathbb{Z}\}
$$

constitute a Bravais lattice. In the three-dimensional space, according to the relationship between the vectors of the basis, we can distinguish 14 types of Bravais lattices which are characterized in Table 5. Notice that they are collected in a set of lattice systems.

\section{A.1.1 Unit Cell}

The space $\mathbb{R}^{3}$ can be subdivided into cells of finite volume having all the same shape. They are called unit cells if these volumes cover all the space without overlapping 
International Journal for Multiscale Computational Engineering, 17(3):261-280 (2019),

Special Issue: Computational Multi-scale Modeling and Design of New Engineering Materials"

Guest Editors: M. Pietrzyk, T.Burczynski, X. Oliver, A. Huespe,)

Table 5: Catalog of the 14 Bravais lattices classified according to their lattice system

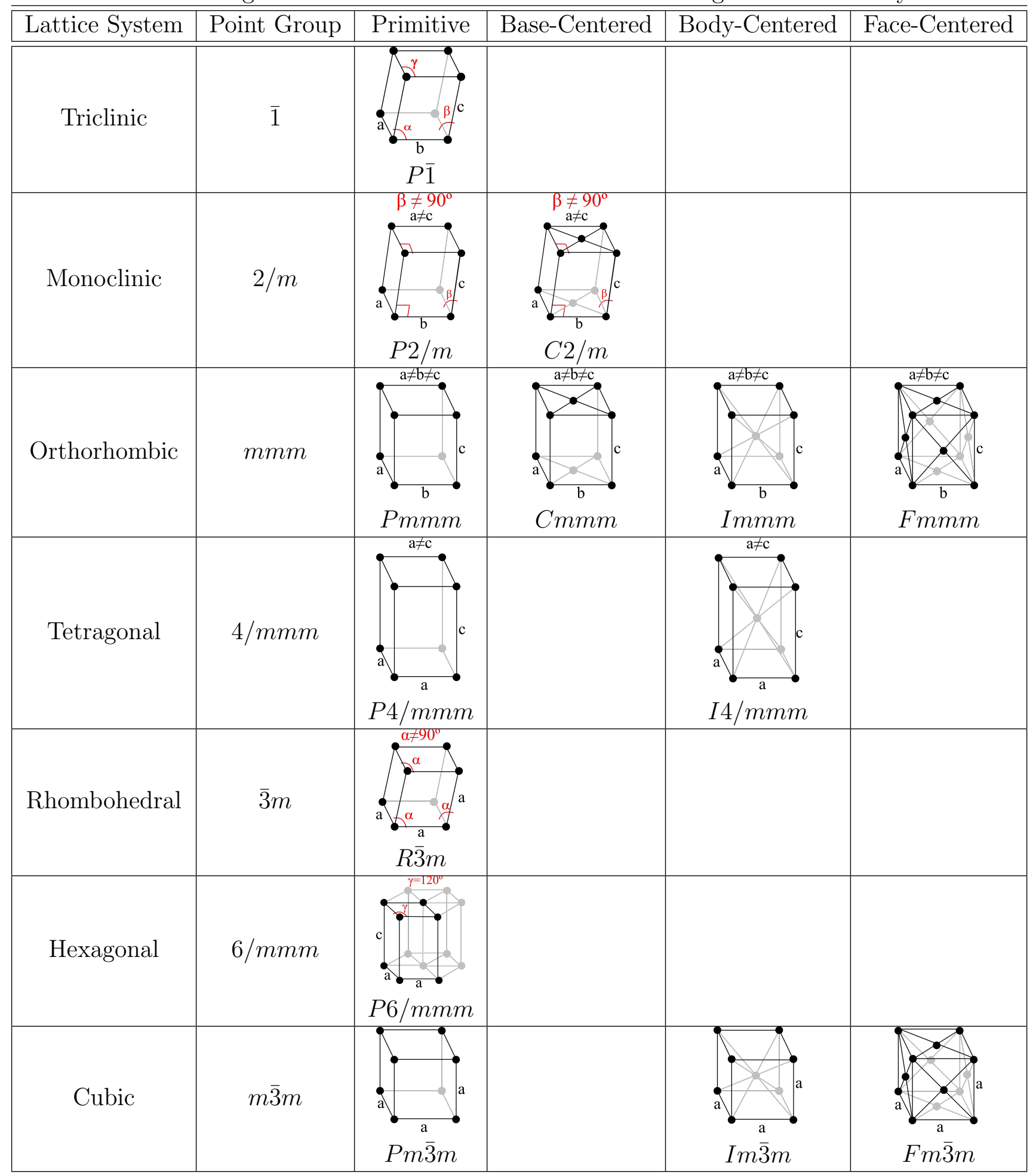


International Journal for Multiscale Computational Engineering, 17(3):261-280 (2019),

Special Issue: Computational Multi-scale Modeling and Design of New Engineering Materials"

Guest Editors: M. Pietrzyk, T.Burczynski, X. Oliver, A. Huespe,)

when are translated by the vectors of the lattice $\mathbf{L}$.

The primitive unit cell is a standard cell that consists of the parallelepiped generated by the primitive vectors of the lattice (third column in Table 5). Although all the 14 Bravais lattices have a primitive unit cell constructed in this way, in many cases, it is more convenient to use a conventional cell in order to better visualize the structure of the lattices (columns 4 to 6 in Table 5). These conventional cells are not necessarily unit cell because they could contain more than one lattice point and consequently their volumes are larger than the volumes of the primitive unit cell.

Another standard construction for the unit cells is the Wigner-Seitz cell. This cell consists of those points of $\mathbb{R}^{3}$ that are closer to a given lattice point than to any other point of $\mathbf{L}$ (see Table 3 for cubic lattices).

Two remarkable features of the Wigner-Seitz cells are noted: $i$ ) its volume is the minimum one that a unit cell can have since, by construction, this cell contains only one lattice atom; ii) it preserves the same point group symmetry of the corresponding Bravais lattice. Primitive unit cells, in general, do not have this property.

\section{A.2 Crystallographic point groups}

Point symmetries are symmetry operations that leave at least one point fixed. There are five types of point symmetries in 3D. They are the inversion ( $\overline{1})$, rotations $(2$, $3,4$ or 6$)$, reflection $(m)$, rotation-inversion $(\overline{2}=m, \overline{3}, \overline{4}$ or $\overline{6})$ and the identity (1). Not all rotations are allowed because they must be compatible with the discrete translation symmetry of the crystals, that is the reason because they are called crystallographic point groups.

After applying the crystallographic restriction theorem, a total of 32 point groups are all the possible symmetries that a given crystal in 3D can have. They are enumerated in the third column of Table 1. By "crystal" we mean a lattice with a base or motif. Not all the point groups are compatible with all lattices, but many of them are compatible with the same group of lattices. This fact allows a classification of point groups in crystal systems, which can be seen in the second column of Table 1.

The lattices themselves (or crystals without a base) have their own point group, which can be seen in the second column of Table 5. Lattices with the same point group are grouped in lattice systems and are shown in the first column of the table.

Unit cells are not unequivocally defined and they can have practically any symmetry. However, as we mentioned above, Wigner-Seitz cells preserve the same symmetry as the associated Bravais lattices, and therefore, they have the same point groups shown in Table 5. As with the lattice, we are considering here Wigner-Seitz cells without a motif in its interior. 
International Journal for Multiscale Computational Engineering, 17(3):261-280 (2019),

Special Issue: Computational Multi-scale Modeling and Design of New Engineering Materials"

Guest Editors: M. Pietrzyk, T.Burczynski, X. Oliver, A. Huespe,)

\section{A.3 Space groups}

On one hand, the discrete translation symmetry of the crystal restricts the infinite possible point groups to only 32 , but, on the other hand, it adds two new kind of symmetries. One type corresponds to the glide planes (denoted by $a, b, c, n$ or $d$ ), which consist of a reflection followed by a translation. The other type corresponds to the screw axis (e.g., $3_{2}, 4_{1}, 6_{3}$ ), which represent a rotation followed by a translation.

Combining these two symmetry types with the 32 point groups, 270 space groups can be classified. In the forth column of Table 1, we can see the amount of them compatible with each crystal and lattice system.

Besides the letters and numbers corresponding to the symmetries, in the nomenclature of space groups also appear a capital letter that helps to identify the compatible Bravais lattice (e.g., $P$ for principal, $F$ for face centered, $I$ for body centered). In Table 5, the space groups of the 14 Bravais lattices can be seen below the images, and in Table 2, the 36 space groups compatible with the 3 cubic lattices are enumerated.

\section{B APPENDIX II: Solving the topology optimiz- ation problem with Topological Derivative Al- gorithm}

We summarize in this Appendix the topology optimization algorithm that is used for solving the numerical test presented in Section 5. The algorithm is a well-established technique reported in the papers by [20] and by [21]. It is a level-set method (LSM) with sensitivity computed through the topological derivative, see [31] and [32]. This technique has been implemented in a 3D code using an Augmented-Lagrangian scheme reported in [33].

Let us introduce a smooth level set-funtion defined in the microcell $\Omega_{\mu}, \psi \in$ $C^{0}\left(\Omega_{\mu}\right)$, satisfying

$$
\psi(\boldsymbol{y})=\left\{\begin{array}{cl}
<0 & \forall \boldsymbol{y} \in \Omega_{\mu}^{2} \\
>0 & \forall \boldsymbol{y} \in \Omega_{\mu}^{1} \\
0 & \text { in the interfaces }
\end{array},\right.
$$

then, the characteristic functions $\chi(\boldsymbol{y})$ in $\Omega_{\mu}$, given by expression (1), can be redefined as follows:

$$
\chi(\psi)=\left\{\begin{array}{ll}
0 & \forall \psi \leq 0 \\
1 & \forall \psi>0
\end{array} .\right.
$$


International Journal for Multiscale Computational Engineering, 17(3):261-280 (2019),

Special Issue: Computational Multi-scale Modeling and Design of New Engineering Materials"

Guest Editors: M. Pietrzyk, T.Burczynski, X. Oliver, A. Huespe,)

and the problem (2) is rephrased as:

$$
\begin{aligned}
\min _{\psi \in C^{0}} & \left\|\boldsymbol{C}^{h}(\psi)-\hat{\boldsymbol{C}}\right\| \\
& \text { such that: } V^{h}(\psi)-V^{o b j}=0 .
\end{aligned}
$$

By making use of an Augmented Lagrangian technique, see [33], the problem (13) is rewritten as follows:

$$
\max _{\lambda} \min _{\psi} \mathcal{T}(\psi, \lambda)
$$

with:

$$
\mathcal{T}(\psi, \lambda)=\left\|\boldsymbol{C}^{h}(\psi)-\hat{\boldsymbol{C}}\right\|+\lambda\left(V^{h}-V^{o b j}\right)+\frac{\alpha}{2}\left(V^{h}-V^{o b j}\right)^{2}
$$

where $V^{h}=\left(\int_{\Omega_{\mu}} \chi(\psi) d \Omega\right) /\left|\Omega_{\mu}\right|$ is the volume fraction of the hard phase, $\lambda$ is the Lagrange multiplier and $\alpha$ is the penalty parameter of the augmented term.

The algorithm for solving the problem (14) has a loop where $\alpha$ is hold fixed and $\lambda$ is modified iteratively.

The minimum of $\mathcal{T}$ is searched with a descent direction algorithm. For problem (14), the descent direction is estimated with the term

$$
D_{\psi} \mathcal{T}(\psi, \lambda)=\frac{\left(\boldsymbol{C}^{h}-\hat{\boldsymbol{C}}\right): D_{\psi} \boldsymbol{C}^{h}}{\left\|\boldsymbol{C}^{h}-\hat{\boldsymbol{C}}\right\|}+\lambda \mathbf{1}+\alpha\left(V^{h}-V^{o b j}\right) \mathbf{1}
$$

where $D_{\psi} \boldsymbol{C}^{h}$ is the topological derivative of the homogenized elasticity tensor and is given by the expressions:

$$
D_{\psi} \boldsymbol{C}_{i j}^{h}=\boldsymbol{\varepsilon}_{\mu}^{i} \cdot \boldsymbol{P} \cdot \boldsymbol{\varepsilon}_{\mu}^{j} \quad ; \quad \boldsymbol{P}=m_{1}\left(m_{2}(\mathbf{1} \otimes \mathbf{1})+2 \mathbb{I}\right) ;
$$

where $\boldsymbol{P}$ is the polarization tensor, $\boldsymbol{\varepsilon}_{\mu}^{i}$ and $\boldsymbol{\varepsilon}_{\mu}^{j}$ are the micro-strain solutions of the homogenization problem solved with the $\mathrm{i}$-th and $\mathrm{j}$-th canonical macro-strains $(i, j=1,2, \ldots, 6)$, the symbols $\mathbf{1}$ and $\mathbb{I}$ represent the second and fourth order unit tensor, respectively, and the coefficient $m_{1}$ and $m_{2}$ are:

$$
\begin{aligned}
m_{1} & =\frac{15 \mu \delta_{\mu}(\nu-1)}{15 \mu(1-\nu)+2 \delta_{\mu}(5 \nu-4)} \\
m_{2} & =\frac{\delta_{\lambda}\left[15 \mu \lambda(1-\nu)+2 \lambda \delta_{\mu}(5 \nu-4)\right]-2 \delta_{\mu}\left(\lambda \delta_{\mu}-5 \mu \nu \delta_{\lambda}\right)}{5 \delta_{\mu}\left[3 \mu \lambda(1-\nu)-3 \mu \nu \delta_{\lambda}-\lambda \delta_{\mu}(1-2 \nu)\right]}
\end{aligned}
$$

with $\delta_{\lambda}=\lambda-\lambda_{0} ; \delta_{\mu}=\mu-\mu_{0}$ and $(\lambda ; \mu)$ being the Lamè parameters of the base (stiff) material and $\left(\lambda_{0} ; \mu_{0}\right)$ being the Lamè parameters of the material introduced as a spherical perturbation. The Poisson ratio of the base material is $\nu$. Additional description and properties of this tensor can be found in [32], where it is called the Elastic Moment Tensor (EMT). 
Then, we define the function:

$$
g(\boldsymbol{y})=\left\{\begin{array}{ll}
-\left(D_{\psi} \mathcal{T}\right) & \text { if }: \psi<0 \\
+\left(D_{\psi} \mathcal{T}\right) & \text { if }: \psi>0
\end{array},\right.
$$

The updating formula for $\psi$ is defined by

$$
\psi^{k+1}=\psi^{k}+\tau g,
$$

with the scaling factor $\tau$ being determined by means of a line search technique.

The Lagrange multiplier $\lambda$ is updated using the Uzawa algorithm

$$
\lambda^{l+1}=\max \left(0, \lambda^{l}+\alpha \int_{\Omega_{\mu}} \chi(\psi) d \Omega\right) .
$$

The penalty parameter $\alpha$ is hold fixed during the full process.

A local optimality criterion of problem (14), see [34], is given by the condition

$$
D_{\psi} \mathcal{T}>0 \quad ; \quad \forall \boldsymbol{y} \in \Omega_{\mu}
$$

which can be implemented by verifying the inequality

$$
\left[\frac{\int_{\Omega_{\mu}} g \psi d V}{\|g\|_{L^{2}}\|\psi\|_{L^{2}}}\right]>\left(1-t o l_{\psi}\right)
$$

combined with

$$
\left\|\boldsymbol{C}^{h}-\hat{\boldsymbol{C}}\right\|<\text { tol }_{C}
$$

he terms $t o l_{\psi}$ and $t o l_{C}$ are tolerances to zero.

Additionally, a Helmholtz-type filter taken from [22] is implemented. The smooth level set function $\tilde{\psi}$, at each $(k+1)$-iteration, is computed by solving the field equation:

$$
r_{l s}^{2} \nabla^{2} \tilde{\psi}^{k+1}+\tilde{\psi}^{k+1}=\psi^{k+1}
$$

with homogeneous boundary conditions $d\left(\tilde{\psi}^{k+1}\right) / d \boldsymbol{n}=0$ on the boundary of $\Omega_{\mu}$. The filter characteristic size $r_{l s}$ determines the minimum length scale in the topology optimization problem.

\section{References}

[1] M.P. Bendsoe and O. Sigmund. Topology optimization: theory, methods, and applications. Springer Science \& Business Media, 2003.

[2] C.G. Méndez, J.M. Podestá, O. Lloberas-Valls, S. Toro, A.E. Huespe, and J. Oliver. Computational material design for acoustic cloaking. International Journal for Numerical Methods in Engineering, 112(10):1353-1380, 2017. 
International Journal for Multiscale Computational Engineering, 17(3):261-280 (2019),

Special Issue: Computational Multi-scale Modeling and

Design of New Engineering Materials"

Guest Editors: M. Pietrzyk, T.Burczynski, X. Oliver, A. Huespe,)

[3] J.M. Podestá, C. Méndez, S. Toro, A.E. Huespe, and J. Oliver. Material design of elastic structures using voronoi cells. International Journal for Numerical Methods in Engineering, 115(3):269-292, 2018.

[4] O. Sigmund. Materials with prescribed constitutive parameters: an inverse homogenization problem. International Journal of Solids and Structures, 31 (17):2313-2329, 1994.

[5] MM Neves, H Rodrigues, and J Miranda Guedes. Optimal design of periodic linear elastic microstructures. Computers \& Structures, 76(1-3):421-429, 2000.

[6] Y. Wang, Z. Luo, N. Zhang, and Z. Kang. Topological shape optimization of microstructural metamaterials using a level set method. Computational Materials Science, 87:178-186, 2014.

[7] P.G. Coelho, L.D. Amiano, J.M. Guedes, and H.C. Rodrigues. Scale-size effects analysis of optimal periodic material microstructures designed by the inverse homogenization method. Computers \& Structures, 174:21-32, 2016.

[8] H. Li, Z. Luo, L. Gao, and P. Walker. Topology optimization for functionally graded cellular composites with metamaterials by level sets. Computer Methods in Applied Mechanics and Engineering, 328:340-364, 2018.

[9] J. Oliver, A. Ferrer, J.C. Cante, S.M. Giusti, and O. Lloberas-Valls. On multiscale computational design of structural materials using the topological derivative. In Advances in Computational Plasticity, pages 289-308. Springer, Cham, 2018.

[10] O. Sigmund. A new class of extremal composites. Journal of the Mechanics and Physics of Solids, 48(2):397-428, 2000.

[11] E. Andreassen and O. Lazarov, B.S.and Sigmund. Design of manufacturable 3d extremal elastic microstructure. Mechanics of Materials, 69(1):1-10, 2014.

[12] A.R. Diaz and A. Benard. Designing materials with prescribed elastic properties using polygonal cells. International Journal for Numerical Methods in Engineering, 57(3):301-314, 2003.

[13] J.M. Podesta, C. Mendez, S. Toro, and A.E. Huespe. Symmetry considerations for topology design in the elastic inverse homogenization problem. Journal of the Mech. Phys. of Solids, 128:54-78, 2019.

[14] O. Sigmund. Tailoring materials with prescribed elastic properties. Mechanics of Materials, 20(4):351-368, 1995. 
International Journal for Multiscale Computational Engineering, 17(3):261-280 (2019),

Special Issue: Computational Multi-scale Modeling and Design of New Engineering Materials"

Guest Editors: M. Pietrzyk, T.Burczynski, X. Oliver, A. Huespe,)

[15] X. Huang, S.W. Zhou, Y.M. Xie, and Q. Li. Topology optimization of microstructures of cellular materials and composites for macrostructures. Computational Materials Science, 67:397-407, 2013.

[16] J. E. Cadman, S. Zhou, Y. Chen, and Q. Li. On design of multi-functional microstructural materials. Journal of Materials Science, 48(1):51-66, 2013.

[17] M. Osanov and J.K. Guest. Topology optimization for architected materials design. Annual Review of Materials Science, 46:211-233, 2016.

[18] F. Feyel and J.L. Chaboche. Fe2 multiscale approach for modelling the elastoviscoplastic behaviour of long fibre sic/ti composite materials. Computer methods in applied mechanics and engineering, 183(3-4):309-330, 2000.

[19] J.C. Michel, H. Moulinec, and P Suquet. Effective properties of composite materials with periodic microstructure: a computational approach. Computer methods in applied mechanics and engineering, 172(1-4):109-143, 1999.

[20] S. Amstutz and H. Andrä. A new algorithm for topology optimization using a level-set method. Journal of Computational Physics, 216(2):573-588, 2006.

[21] S. Amstutz, S.M. Giusti, A.A. Novotny, and E.A. de Souza Neto. Topological derivative for multi-scale linear elasticity models applied to the synthesis of microstructures. International Journal for Numerical Methods in Engineering, 84(6):733-756, 2010.

[22] B. S. Lazarov and O. Sigmund. Filters in topology optimization based on helmholtz-type differential equations. Int. J. Numer. Meth. Engng, 86:765781, 2011.

[23] F. Wang, B. S. Lazarov, and O. Sigmund. On projection methods, convergence and robust formulations in topology optimization. Structural and Multidisciplinary Optimization, 43(6):767-784, 2011.

[24] J. Sólyom. Fundamentals of the Physics of Solids: Volume 1: Structure and Dynamics, volume 1. Springer Science \& Business Media, 2007.

[25] B. Souvignier. A general introduction to space groups. International Tables for Crystallography, A:22-41, 2016. Chapter:1.3.

[26] J.F. Nye. Physical Properties of Crystals: Theri representation be tensors and matrices, volume 146. Clarendon Press- Oxford, 2006.

[27] T.C. Ting. Anisotropic elasticity: theory and applications. Number 45. Oxford University Press, 1996. 
International Journal for Multiscale Computational Engineering, 17(3):261-280 (2019),

Special Issue: Computational Multi-scale Modeling and

Design of New Engineering Materials"

Guest Editors: M. Pietrzyk, T.Burczynski, X. Oliver, A. Huespe,)

[28] X. Huang, A. Radman, and Y.M. Xie. Topological design of microstructures of cellular materials for maximum bulk or shear modulus. Computational Materials Science, 50(6):1861-1870, 2011.

[29] Z. Hashin and S. Shtrikman. A variational approach to the theory of the elastic behaviour of multiphase materials. Journal of the Mechanics and Physics of Solids, 11(2):127-140, 1963.

[30] S. Meille and E.J. Garboczi. Linear elastic properties of $2 \mathrm{~d}$ and 3d models of porous materials made from elongated objects. Modelling and Simulation in Materials Science and Engineering, 9(5):371, 2001.

[31] A.A. Novotny and J. Sokołowski. Topological derivatives in shape optimization. Springer Science \& Business Media, 2012.

[32] H. Ammari, P. Calmon, and E. Iakovleva. Direct elastic imaging of a small inclusion. SIAM Journal on Imaging Sciences, 1(2):169-187, 2008.

[33] C.G. Lopes, R.B. dos Santos, and A.A. Novotny. Topological derivative-based topology optimization of structures subject to multiple load-cases. Latin American Journal of Solids and Structures, 12(5):834-860, 2015.

[34] S. Amstutz. Analysis of a level set method for topology optimization. Optimization Methods and Software, 26(4-5):555-573, 2011. 